\title{
von Neumann stability conditions for the convection-diffusion equation
}

\author{
P. WESSELING \\ Delft University of Technology, Faculty of Technical Mathematics and Informatics, \\ PO Box 5031, 2600 GA Delft. The Netherlands
}

[Received 25 May 1995 and in revised form 13 November 1995]

\begin{abstract}
A method is presented to easily derive von Neumann stability conditions for a wide variety of time discretization schemes for the convection-diffusion equation. Spatial discretization is by the $\kappa$-scheme or the fourth-order central scheme. The use of the method is illustrated by application to multistep, Runge-Kutta and implicit-explicit methods, such as are in current use for flow computations, and for which, with few exceptions, no sufficient von Neumann stability results are available.
\end{abstract}

\section{Introduction}

Stability criteria for the non-stationary convection-diffusion equation often result immediately in stability conditions for numerical methods to compute nonstationary incompressible flows, if the widely used pressure-correction method (Chorin (1968), van Kan (1986)) is applied. This is because for the velocity prediction step something very close to the convection-diffusion equation is solved, and the stability analysis boils down to the stability analysis for the convection-diffusion equation, which is the topic of this paper. Here, by stability we mean stability in the sense of von Neumann, i.e. non-growth of Fourier components in the frozen coefficients case on an unbounded domain. Strictly speaking, an $\mathrm{O}(\Delta t)$ growth per time step could be allowed, but this hardly affects the stability conditions that result.

We will present a method that is both simple and applicable to a wide variety of schemes and that gives stability conditions that are easily evaluated and not too conservative. The method has been outlined before in Wesseling (1995) for central discretization, using a second-or fourth-order scheme for the convection term. Here the $\kappa$-scheme (van Leer $(1985)$ ) or the first-order upwind scheme will be used for the convection term. Time discretizations will-be considered that are often employed for non-stationary incompressible flow computations, such as large-eddy or direct simulation of turbulence. No rigorous von Neumann stability conditions seem to have been derived before for these schemes.

Our approach is as follows. In Section 2 we recall that for von Neumann stability it is necessary that the symbol $\hat{L}_{h}$ of the discrete operator is inside the stability domain $S$ of the time discretization method. In Section 3 we derive conditions for $\hat{L}_{h}$ to be inside certain simple geometric figures. In Section 4 we 
fit these figures in $S$ to a number of illustrative examples, obtaining explicit sufficient stability conditions, and showing that the method is quite general and easy to apply.

\section{Fourier stabllity analysis}

The convection-diffusion equation is given by

$$
\frac{\partial \varphi}{\partial t}+L \varphi=0, \quad L \varphi=\sum_{\alpha=1}^{m}\left(u_{\alpha} \frac{\partial}{\partial x_{\alpha}}-\nu \frac{\partial^{2}}{\partial x_{\alpha}^{2}}\right) \varphi
$$

with $m$ the number of dimensions. For Fourier stability analysis, $u_{\alpha}$ and $v$ are taken constant, and the domain is unbounded. With the $\kappa$-scheme, the discretization of $L$ on a uniform grid with mesh-sizes $h_{1}, \ldots, h_{m}$ is given by $L_{h}=C_{h}+D_{h}$ with

$$
C_{h}=\frac{1}{4 \tau} \sum_{\alpha} c_{\alpha}\left\{(1-\kappa) \varphi_{j-2 e_{\alpha}}-(5-3 \kappa) \varphi_{j-e_{\alpha}}+(3-3 \kappa) \varphi_{j}+(1+\kappa) \varphi_{j+e_{\alpha}}\right\}
$$

and

$$
D_{h}=\frac{1}{2 \tau} \sum_{\alpha} d_{\alpha}\left(-\varphi_{j-e_{\alpha}}+2 \varphi_{j}-\varphi_{j+e_{\alpha}}\right)
$$

where $j=\left(j_{1}, \ldots, j_{m}\right), e_{1}=(1,0, \ldots, 0), e_{2}=(0,1,0, \ldots, 0)$, etc, and

$$
c_{\alpha}=u_{\alpha} \tau / h_{\alpha}, \quad d_{\alpha}=2 \nu \tau / h_{\alpha}^{2}
$$

with $\tau$ the time step. The dimensionless numbers $c_{\alpha}$ and $d_{\alpha}$, called the Courant and diffusion numbers, respectively, govern stability. In (2.2), $u_{\alpha} \geqslant 0$ has been assumed; the contrary case may be treated by symmetry. For $\kappa=-1$ we have the one-sided fully upwind scheme (Steger \& Warming (1981)). With $\kappa=0$ one obtains Fromm's zero average phase error scheme (Fromm (1968)), if the terms quadratic in the Courant number are neglected, which are meant to improve time accuracy; Fromm's scheme is the result of optimizing, among 5-point schemes, for the propagation of a step function over one time step in the absence of diffusion (Wesseling (1973)). For $k=1 / 3$ the third-order upwind biased scheme (Anderson et al (1985)) results. For $k=1 / 2$ we have the second-order QUICK (quadratic upstream interpolation for convective kinematics) scheme proposed in Leonard (1979). Finally, $k=1$ gives the central second-order scheme.

A fourth-order central discretization of the convection term is given by

$$
C_{h}=\frac{1}{12 \tau} \sum_{\alpha} c_{\alpha}\left(\varphi_{j-2 e_{\alpha}}-8 \varphi_{j-e_{a}}+8 \varphi_{j+e_{\alpha}}-\varphi_{j+2 e_{a}}\right)
$$

First-order upwind discretization is included in the analysis that follows by taking $\kappa=1$ and redefining $d_{\alpha}=d_{\alpha}+\left|c_{\alpha}\right|$.

After spatial discretization we are left with the following system of ordinary differential equations:

$$
\mathrm{d} \varphi / \mathrm{d} t=-L_{h} \varphi_{j}
$$


The symbol or Fourier transform $\hat{L}_{h}(\theta)$ of $L_{h}$ is defined by $\hat{L}_{h}(\theta)=\mathrm{e}^{-\mathrm{i} j \theta} L_{h} \mathrm{e}^{\mathrm{i} j \theta}$ with $\theta=\left(\theta_{1}, \ldots, \theta_{m}\right)$. One finds:

$$
\begin{aligned}
\tau \hat{L}_{h}(\theta) & =\hat{C}_{h}(\theta)+\hat{D}_{h}(\theta) \\
\hat{C}_{h}(\theta) & =\gamma_{1}(\theta)+\mathrm{i} \gamma_{2}(\theta), \quad \hat{D}_{h}(\theta)=\delta(\theta) .
\end{aligned}
$$

For (2.2) we have

$$
\gamma_{1}(\theta)=2(1-\kappa) \sum_{\alpha}\left|c_{\alpha}\right| s_{\alpha}^{2}, \quad \gamma_{2}(\theta)=\sum_{\alpha} c_{\alpha}\left\{(1-\kappa) s_{\alpha}+1\right\} \sin \theta_{\alpha}
$$

where $s_{\alpha}=\sin ^{2} \frac{1}{2} \theta_{\alpha}$, whereas for (2.5)

$$
\gamma_{1}(\theta)=0, \quad \gamma_{2}(\theta)=\frac{1}{6} \sum_{\alpha} c_{\alpha}\left(8 \sin \theta_{\alpha}-\sin 2 \theta_{\alpha}\right) .
$$

Furthermore,

$$
\delta(\theta)=2 \sum_{\alpha} d_{\alpha} s_{\alpha}
$$

For Fourier (or von Neumann) stability analysis one substitutes $\varphi_{j}(t)=y(t) \mathrm{e}^{\mathrm{i} j \theta}$ in (2.6), and obtains

$$
\mathrm{d} y / \mathrm{d} t=-\hat{L}_{h}(\theta) y .
$$

Sufficient for von Neumann stability is

$$
S_{L} \subseteq S, \quad S_{L}=\left\{-\tau \hat{L}_{h}(\theta) \in \mathbb{C}: \forall \theta\right\}
$$

with $S$ the stability domain of the time discretization method to be used.

\section{Some useful theorems}

For the derivation of sufficient stability conditions, the following theorems, which form the substance of our method, are useful. But first some preliminaries. The $\kappa$-scheme (2.2), (2.3) will be called scheme 1 and (2.3), (2.5) will be called scheme 2. Define $\tilde{c}_{\alpha}=(1-\kappa)\left|c_{\alpha}\right|$ for scheme 1 and $\tilde{c}_{\alpha}=0$ for scheme 2. Let

$$
\tilde{d}_{\alpha}=d_{\alpha}+\tilde{c}_{\alpha} \quad \text { and } \quad \tilde{d}=\sum_{\alpha} \tilde{d}_{\alpha} .
$$

Schwarz's inequality will be used frequently. For schemes 1 and 2 we have

$$
\left(\delta+\gamma_{1}\right)^{2}=4\left\{\sum_{\alpha} d_{\alpha} s_{\alpha}+\tilde{c}_{\alpha} s_{\alpha}^{2}\right\}^{2} \leqslant 4\left\{\sum_{\alpha} \tilde{d}_{\alpha} s_{\alpha}\right\}^{2} \leqslant 4 \tilde{d} \sum_{\alpha} \tilde{d}_{\alpha} s_{\alpha}^{2}
$$

Furthermore, for scheme 1,

$$
\gamma_{2}^{2} \leqslant 4 \sum_{\alpha}\left(c_{\alpha}^{2} / d_{\alpha}\right) \sum_{\alpha} d_{\alpha} s_{\alpha}\left(1-s_{\alpha}\right)\left(\bar{k} s_{\alpha}+1\right)^{2}
$$


where $\bar{\kappa}=1-\kappa$, whereas for scheme 2 we find that (3.2) holds with $\kappa=1 / 3$. From (3.2) it follows that

$$
\gamma_{2}^{2} \leqslant 4(2-\kappa)^{2} \sum_{\alpha}\left(c_{\alpha}^{2} / d_{\alpha}\right) \sum_{\alpha} d_{\alpha} s_{\alpha}\left(1-s_{\alpha}\right)
$$

Similarly, for scheme 1 ,

$$
\begin{aligned}
\gamma_{2}^{4} & \leqslant\left\{\sum_{\alpha}\left(\left|c_{\alpha}\right|^{2 / 3} d_{\alpha}^{-1 / 6}\right)\left|c_{\alpha}\right|^{1 / 3} d_{\alpha}^{1 / 6}\left(\bar{\kappa} s_{\alpha}+1\right)\left|\sin \theta_{\alpha}\right|\right\}^{4} \\
& \leqslant 16\left\{\sum_{\alpha}\left(c_{\alpha}^{4} / d_{\alpha}\right)^{1 / 3}\right\}^{3} \sum_{\alpha} d_{\alpha}\left(\bar{\kappa} s_{\alpha}+1\right)^{4} s_{\alpha}^{2}\left(1-s_{\alpha}\right)^{2}
\end{aligned}
$$

which also holds for scheme 2 with $k=1 / 3$. For arbitrary $a>0$ we have for schemes 1 and 2

$$
\left(\frac{\delta+\gamma_{1}}{a}-1\right)^{2} \leqslant 1+\frac{4 \tilde{d}}{a^{2}} \sum_{\alpha} \tilde{d}_{\alpha} s_{\alpha}^{2}-\frac{4}{a} \sum_{\alpha}\left(d_{\alpha} s_{\alpha}+\tilde{c}_{\alpha} s_{\alpha}^{2}\right)
$$

If $\tilde{d} \leqslant a$ this gives

$$
\left(\frac{\delta+\gamma_{1}}{a}-1\right)^{2} \leqslant 1+\frac{4}{a} \sum_{\alpha} d_{\alpha}\left(s_{\alpha}^{2}-s_{\alpha}\right)
$$

THEOREM 3.1 If

$$
\tilde{d} \leqslant a \quad \text { and } \quad \sum_{\alpha} c_{\alpha}^{2} / d_{\alpha} \leqslant(2-\kappa)^{-2} b^{2} / a
$$

then for scheme $1 S_{L}$ is contained in the ellipse

$$
(v / a+1)^{2}+(w / b)^{2}=1, \quad v+\mathrm{i} w=z .
$$

The first condition is necessary.

Proof. Necessity of the first condition follows by taking $s_{\alpha}=1, \alpha=1, \ldots, m$. It remains to show that $\left\{\left(\delta+\gamma_{1}\right) / a-1\right\}^{2}+\left(\gamma_{2} / b\right)^{2} \leq 1$. Using (3.3), (3.6) and (3.7) we have

$$
\left(\frac{\delta+\gamma_{1}}{a}-1\right)^{2}+\left(\frac{\gamma_{2}}{b}\right)^{2} \leqslant 1+\frac{4}{a} \sum_{\alpha} d_{\alpha} s_{\alpha}\left(1-s_{\alpha}\right)(-1+1) \leqslant 1
$$

and the proof is complete.

For $\kappa=1$ necessity of both conditions is shown in Wesseling (1995). The conditions (3.7) are equivalent to

$$
\tau \leqslant \min \left\{a / \sum_{\alpha}\left(2 v h_{\alpha}^{-2}+(1-\kappa)\left|u_{\alpha}\right| h_{\alpha}^{-1}\right), 2 v(2-\kappa)^{-2} \frac{b^{2}}{a} / \sum_{\alpha} u_{\alpha}^{2}\right\} .
$$


THEOREM 3.2 If

$$
\tilde{d} \leqslant a
$$

and one or both of the following two conditions hold:

$$
\sum_{\alpha}\left(c_{\alpha}^{4} / d_{\alpha}\right)^{1 / 3} \leqslant q_{1}\left(b^{4} / a\right)^{1 / 3} \quad \text { or } \quad \sum_{\alpha}\left|c_{\alpha}\right| \leqslant q_{2} b^{2} / a
$$

where

$$
\begin{aligned}
q_{1} & =\frac{1}{4}(8-4 \kappa)^{-5 / 3}(15-5 \kappa-r)^{4 / 3}(5 \kappa-3+r)^{1 / 3}(9-7 \kappa+r)^{1 / 3}, \\
r & =\left(25 \kappa^{2}-54 \kappa+33\right)^{1 / 2}, \\
q_{2} & = \begin{cases}(1-\kappa)^{3 / 2}(8 / 5-4 \kappa / 5)^{-5 / 2}, & -1 \leqslant \kappa<1 / 2, \\
(1-\kappa) / 2, & 1 / 2 \leqslant \kappa \leqslant 1\end{cases}
\end{aligned}
$$

then for scheme $1 S_{L}$ is contained in the oval given by

$$
(v / a+1)^{2}+(w / b)^{4}=1, \quad v+\mathrm{i} w=z .
$$

Condition (3.10) is necessary.

Proof. Necessity of (3.10) follows by taking $s_{\alpha}=1, \alpha=1 \ldots . m$. Next, assume that (3.10) and the first condition of (3.11) hold. Using (3.4) and (3.6),

$$
\left(\frac{\delta+\gamma_{1}}{a}-1\right)^{2}+\left(\frac{\gamma_{2}}{b}\right)^{4} \leqslant 1+\frac{4}{a} \sum_{\alpha} d_{\alpha}\left(s_{\alpha}-s_{\alpha}^{2}\right)\left\{-1+4 q_{1}^{3}\left(\bar{\kappa} s_{\alpha}+1\right)^{4} s_{\alpha}\left(1-s_{\alpha}\right)\right\}
$$

We have

$$
\max \left\{(\bar{\kappa} s+1)^{4} s(1-s): 0 \leqslant s \leqslant 1\right\}=1 / 4 q_{1}^{3} .
$$

Hence, no term in the sum in (3.13) is positive, so that

$$
\left(\frac{\delta+\gamma_{1}}{a}-1\right)^{2}+\left(\frac{\gamma_{2}}{b}\right)^{4} \leqslant 1
$$

Next, assume that (3.10) and the second condition of (3.11) hold. Because of (3.10), $0 \leqslant \delta+\gamma_{1} \leqslant 2 a$, hence

$$
\begin{aligned}
\left(\frac{\delta+\gamma_{1}}{a}-1\right)^{2} & =\frac{\delta}{a}\left(\frac{\delta+\gamma_{1}}{a}+\frac{\gamma_{1}}{a}-2\right)+\left(\frac{\gamma_{1}}{a}-1\right)^{2} \\
& \leqslant\left(\frac{\delta}{a}-2\right) \frac{\gamma_{1}}{a}+\left(\frac{\gamma_{1}}{a}\right)^{2}+1 \\
& \leqslant\left(2 \frac{\tilde{d}}{a}-2-\frac{2}{a} \sum_{\alpha} \tilde{c}_{\alpha}\right) \frac{2}{a} \sum_{\alpha} \tilde{c}_{\alpha} s_{\alpha}^{2}+\frac{4}{a^{2}} \sum_{\alpha} \tilde{c}_{\alpha} \sum_{\alpha} \tilde{c}_{\alpha} s_{\alpha}^{4}+1 \\
& \leqslant 1-\frac{4 \tilde{c}}{a^{2}} \sum_{\alpha} \tilde{c}_{\alpha}\left(s_{\alpha}^{2}-s_{\alpha}^{4}\right)
\end{aligned}
$$


where $\tilde{c}=\sum_{\alpha} \tilde{c}_{\alpha}$. Furthermore, similar to (3.4),

$$
\begin{aligned}
\gamma_{2}^{4} & \leqslant 16\left\{\sum_{\alpha}\left(c_{\alpha}^{4} / \tilde{c}_{\alpha}\right)^{1 / 3}\right\}^{3} \sum_{\alpha} \tilde{c}_{\alpha}\left(\bar{\kappa} s_{\alpha}+1\right)^{4} s_{\alpha}^{2}\left(1-s_{\alpha}\right)^{2} \\
& =\frac{16}{\bar{\kappa}}\left\{\sum_{\alpha}\left|c_{\alpha}\right|\right\}^{3} \sum_{\alpha} \tilde{c}_{\alpha}\left(\bar{\kappa} s_{\alpha}+1\right)^{4} s_{\alpha}^{2}\left(1-s_{\alpha}\right)^{2}
\end{aligned}
$$

Hence

$$
\begin{aligned}
\left(\frac{\delta+\gamma_{1}}{a}-1\right)^{2}+\left(\frac{\gamma_{2}}{b}\right)^{4} \leqslant & +\frac{4 \tilde{c}}{a^{2}} \sum_{\alpha} \tilde{c}_{\alpha} s_{\alpha}^{2}\left(1-s_{\alpha}\right) \\
& \times\left\{\frac{4 q_{2}^{2}}{\bar{\kappa}^{2}}\left(\bar{\kappa} s_{\alpha}+1\right)^{4}\left(1-s_{\alpha}\right)-s_{\alpha}-1\right\} .
\end{aligned}
$$

Observing that

$$
\max \left\{(\bar{\kappa} s+1)^{4}(1-s) /(1+s): 0 \leqslant s \leqslant 1,0 \leqslant \bar{\kappa} \leqslant 1 / 2\right\}=1
$$

and that

$$
\max \left\{(\bar{\kappa} s+1)^{4}(1-s): 0 \leqslant s \leqslant 1,1 / 2<\bar{\kappa} \leqslant 2\right\}=\bar{\kappa}^{2} / 4 q_{2}^{2}
$$

we see that each term in the preceding sum is non-positive, hence (3.14) holds, and the proof is complete.

Note that the first condition of (3.11) is not useful in the hyperbolic case ( $\nu=0$, i.e. $d_{\alpha}=0$ ), whereas the second condition is not useful for $\kappa=1$; the two conditions complement each other.

Conditions (3.10) and (3.11) are equivalent to

$$
\tau \leqslant a / \sum_{\alpha}\left(2 v h_{\alpha}^{-2}+(1-\kappa)\left|u_{\alpha}\right| h_{\alpha}^{-1}\right)
$$

and

$$
\tau \leqslant \max \left\{q_{1}\left(\frac{2 v b^{4}}{a}\right)^{1 / 3} / \sum_{\alpha}\left(u_{\alpha}^{4} / h_{\alpha}^{2}\right)^{1 / 3}, \frac{q_{2} b^{2}}{a} / \sum_{\alpha}\left|u_{\alpha}\right| h_{\alpha}^{-1}\right\} .
$$

THEOREM 3.3 If

$$
\tilde{d} \leqslant \frac{a}{2} \quad \text { and } \quad \frac{2 \tilde{d}}{b^{2}} \sum_{\alpha} c_{\alpha}^{2} / \tilde{d}_{\alpha} \leqslant(2-\kappa)^{-2}\left(1+\sqrt{1-4 \tilde{d}^{2} / a^{2}}\right)
$$

then for scheme $1 S_{L}$ is contained in the ellipse given by

$$
(v / a)^{2}+(w / b)^{2}=1, \quad v+\mathrm{i} w=z .
$$

The first condition is necessary. 
Proof. Necessity of the first condition follows by taking $s_{\alpha}=1, \alpha=1, \ldots, m$. Using (3.1), (3.3) and (3.16) we have

$$
\left(\frac{\delta+\gamma_{1}}{a}\right)^{2}+\left(\frac{\gamma_{2}}{b}\right)^{2} \leqslant 4 \frac{\tilde{d}}{a^{2}} \sum_{\alpha} \tilde{d}_{\alpha} s_{\alpha}\left\{s_{\alpha}+p\left(1-s_{\alpha}\right)\right\}
$$

where

$$
p=\left(a^{2} / 2 \tilde{d}^{2}\right)\left(1+\sqrt{1-4 \tilde{d}^{2} / a^{2}}\right) .
$$

Since $p>2$,

$$
\max \{s(s+p(1-s)): 0 \leqslant s \leqslant 1\}=p^{2} /\{4(p-1)\}=a^{2} /\left(4 \bar{d}^{2}\right)
$$

so that

$$
\left(\frac{\delta+\gamma_{1}}{a}\right)^{2}+\left(\frac{\gamma_{2}}{b}\right)^{2} \leqslant 1
$$

and the proof is complete.

The conditions (3.16) are equivalent to

$$
\begin{aligned}
\tau \leqslant & \min \left[\frac{1}{2} a / \sum_{\alpha}\left(2 \nu h_{\alpha}^{-2}+(1-\kappa)\left|u_{\alpha}\right| h_{\alpha}^{-1}\right)\right. \\
& b(2-\kappa)^{-1}\left[\sum_{\alpha}\left\{2 \nu h_{\alpha}^{-2}+(1-\kappa)\left|u_{\alpha}\right| h_{\alpha}^{-1}\right\}\right]^{-1 / 2} \\
& \left.\times\left[\sum_{\alpha} u_{\alpha}^{2} /\left\{v+\frac{1}{2}(1-\kappa)\left|u_{\alpha}\right| h_{\alpha}\right\}\right]^{-1 / 2}\left[1+\sqrt{1-4 \tilde{d}^{2} / a^{2}}\right]^{1 / 2}\right] .
\end{aligned}
$$

This inequality is implicit, because $\tilde{d}$ depends on $\tau$. However, checking the admissibility of a given $\tau$ is straightforward, whereas generation of a suitable $\tau$ is easily done by some iterative process.

THEOREM 3.4 If

$$
\sum_{\alpha} c_{\alpha}^{2} / d_{\alpha} \leqslant q_{3} b^{2}
$$

where

$$
q_{3}=\left\{\begin{array}{lll}
1 / 2 & \text { if } & 1 / 2 \leqslant \kappa \leqslant 1, \\
\frac{27}{8}(1-\kappa) /(2-\kappa)^{3} & \text { if } & -1 \leqslant \kappa<1 / 2
\end{array}\right.
$$

then for scheme $1 S_{L}$ is contained in the parabola given by

$$
v+(w / b)^{2}=0, \quad v+\mathrm{i} w=z .
$$


Proof. We have to show that $-\delta-\gamma_{1}+\left(\gamma_{2} / b\right)^{2} \leqslant 0$. Using (3.2) we have

$$
-\delta-\gamma_{1}+\left(\gamma_{2} / b\right)^{2} \leqslant 2 \sum_{\alpha} d_{\alpha} s_{\alpha}\left\{-1+2 q_{3}\left(1-s_{\alpha}\right)\left(\bar{\kappa} s_{\alpha}+1\right)^{2}\right\} .
$$

Observing that

$$
\max \left\{(1-s)(\bar{\kappa} s+1)^{2}: 0 \leqslant s \leqslant 1\right\}=1 / 2 q_{3}
$$

the proof is complete.

Condition (3.19) is equivalent to

$$
\tau \leqslant 2 v q_{3} b^{2} / \sum_{\alpha}\left|u_{\alpha}\right|^{2}
$$

For scheme 2 we have $\gamma_{1}=0$ and (3.2) holds with $\kappa=1 / 3$. Hence, it is easy to see that Theorems $3.1,3.3$ and 3.4 hold with $\tilde{d}$ and $\tilde{d}_{\alpha}$ replaced by $d=\sum_{\alpha} d_{\alpha}$ and $d_{\alpha}$, and $\kappa=1 / 3$, whereas instead of Theorem 3.2 we have

THEOREM 3.5 If

$$
d \leqslant a \quad \text { and } \quad \sum_{\alpha}\left(c_{\alpha}^{4} / d_{\alpha}\right)^{1 / 3} \leqslant q_{4}\left(b^{4} / a\right)^{1 / 3}
$$

with

$$
q_{4}=\frac{1}{5}(\sqrt{10}-1)^{5 / 3}\left(\frac{5+\sqrt{10}}{12}\right)^{1 / 3}
$$

then for scheme $2 S_{L}$ is contained in the oval given by (3.12). The first condition of (3.25) is necessary.

Proof. Necessity of the first part of (3.25) follows by taking $s_{\alpha}=1, \alpha=1, \ldots, m$. The remainder of the proof is the same as the proof showing that (3.10) and the first part of (3.11) are sufficient, substituting $\gamma_{1}=0$ and $\kappa=1 / 3$. $\tau$ :

Conditions (3.25) are equivalent to the following restrictions on the time step

$$
\tau \leqslant \min \left\{\frac{a}{2 v} / \sum_{\alpha} h_{\alpha}^{-2}, q_{4}\left(\frac{2 v b^{4}}{a}\right)^{1 / 3} / \sum_{\alpha}\left(u_{\alpha}^{4} / h_{\alpha}^{2}\right)^{1 / 3}\right\} .
$$

\section{4. von Neumann stability conditions for various time discretizations}

All that remains to be done is to choose suitable unions or intersections of the sets considered in Theorems 3.1-3.5 (ellipses, oval, parabola) inside the stability domain $S$ of the time discretization method, and useful (i.e. not too conservative and easy to evaluate) sufficient conditions for von Neumann stability tumble like ripe apples. It appears that until now only in rare cases have sufficient von Neumann stability conditions been published for the explicit or implicitexplicit (IMEX: diffusion implicit, convection explicit) schemes currently used for 


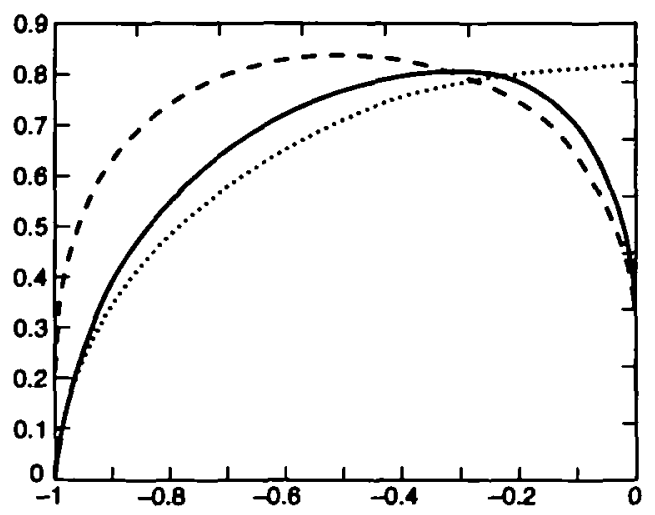

FIG. 1. Stability domain of the second-order Adams-Bashforth scheme (-) with oval (- - -) and ellipse (· • $)$.

time-dependent incompressible flow computations. In some cases necessary but not sufficient conditions are known for the Courant numbers $c_{\alpha}$. Fully implicit schemes are usually easily seen to be unconditionally stable. The techniques used to derive the preceding theorems can also be used to obtain stability conditions for cases where the ellipses, oval and/or parabola do not fit nicely in $S$; an example will be given. Applications will be given to schemes of current or historical interest.

Explicit Euler The stability domain $S$ is the disk $|z+1| \leqslant 1$. With $a=b=1$, Theorem 3.1 immediately gives sufficient stability conditions. In the case $\kappa=1$ (second-order central scheme) these conditions are shown to be also necessary in Wesseling (1995), and identical to those obtained in Hirt (1968), Morton (1971), Hindmarsh et al (1984).

Adams-Bashforth The second-order Adams-Bashforth scheme has the following characteristic polynomial:

$$
\xi^{2}-\xi+z\left(\frac{3}{2} \xi-\frac{1}{2}\right)
$$

with $z=\delta+\gamma_{1}+\mathrm{i} \gamma_{2}$. The boundary $\partial S$ of the stability domain $S$ is found by substituting $\xi=\mathrm{e}^{\mathrm{i} \mu}, 0 \leqslant \mu<2 \pi$ and solving for $-z$; the result is shown in Fig. 1. Only the upper half of $S$ is shown; all the stability domains to be encountered are symmetric with respect to the real axis. Near $z=0$ we have $|\mu| \ll 1$, and on $\partial S$ we have $w \cong \pm(-4 v)^{1 / 4}, v+\mathrm{i} w=z$. On the oval (3.12) we have $w \cong \pm b(-2 v / a)^{1 / 4} ; a=1 / 2$ and $b=2^{-1 / 4}$ result in the oval shown in Fig. 1. Near $z=-1$ we have $\mu=\pi+\varepsilon,|\varepsilon| \ll 1$, and on $\partial S: z \cong-1+v+\mathrm{i} w$, $w=3 \sqrt{v / 5}$. On the ellipse (3.17) with $a=1$ we have $z \cong-1+v+\mathrm{i} w$ with $w=b \sqrt{2 v}$. Choosing $b=\sqrt{2 / 3}$ results in the ellipse shown in Fig. 1. Useful sufficient stability conditions are obtained by requiring $-\tau \hat{L}_{k}(\theta) \subseteq$ ellipse $\cap$ oval. 


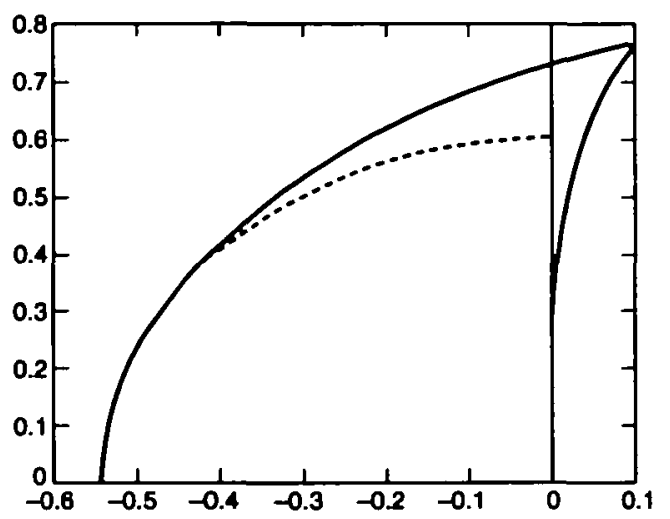

FIG. 2. Stability domain of the third-order Adams-Bashforth scheme ( $\rightarrow$ with osculating ellipse $(--)$.

Combining Theorems 3.2 and 3.3 results in the following sufficient conditions for von Neumann stability for scheme 1:

$$
\tilde{d} \leqslant 1 / 2 \quad \text { and } \quad 3 \tilde{d} \sum_{\alpha} c_{\alpha}^{2} / \tilde{d}_{\alpha} \leqslant(2-\kappa)^{-2}\left(1+\sqrt{1-4 \tilde{d}^{2}}\right)
$$

and

$$
\left\{\sum_{\alpha}\left(c_{\alpha}^{4} / d_{\alpha}\right)^{1 / 3} \leqslant q_{1} \quad \text { or } \quad \sum_{\alpha}\left|c_{\alpha}\right| \leqslant q_{2} \sqrt{2}\right\} .
$$

The corresponding restrictions on $\tau$ are easily found from (3.15) and (3.18) by substitution of the relevant values for $a$ and $b$. Similar conditions can be obtained for scheme 2 by using Theorem 3.5 instead of Theorem 3.2. The third-order Adams-Bashforth scheme has the following characteristic polynomial:

$$
\xi^{3}-\xi^{2}+\frac{1}{12} z\left(23 \xi^{2}-16 \xi+5\right) \text {. }
$$

The stability domain is given in Fig. 2. It is covered to a satisfactory extent by the ellipse (3.17) that osculates in $z=-6 / 11$; its parameters are found to be

$$
a=6 / 11, \quad b=\frac{72}{11} \sqrt{\frac{2}{235}} \cong 0.6038 .
$$

Theorem 3.3 immediately results in sufficient von Neumann stability conditions.

Runge-Kutta To show the versatility of the method, we also apply it to a Runge-Kutta method. For a four-stage method, the amplification factor is given by

$$
P(z)=1+z\left(1+\alpha_{3} z\left(1+\alpha_{2} z\left(1+\alpha_{1} z\right)\right)\right)
$$




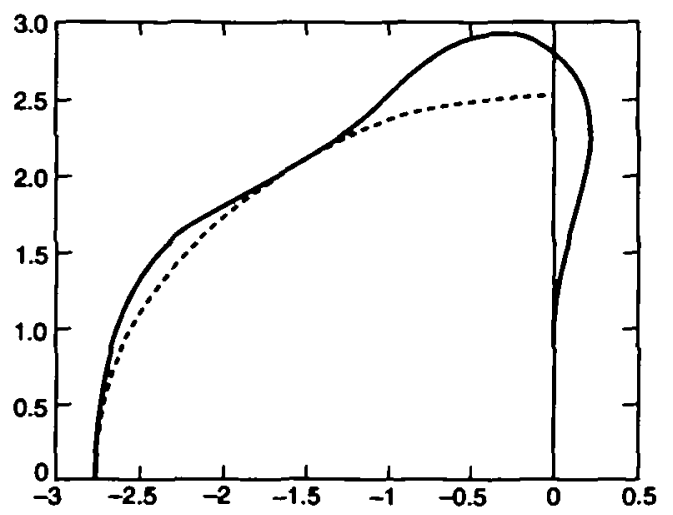

FIG. 3. Stability domain of the RK24 scherne (-) and ellipse (- - -).

In Sommeijer et al (1994) a Runge-Kutta method is presented that is especially designed for convection-diffusion problems, with coefficients $\alpha_{1}=1 / 4, \alpha_{2}=1 / 3$, $\alpha_{3}=1 / 2$. For von Neumann stability we must have

$$
|P(z)| \leqslant 1 \quad \text { for } \quad-z \in S_{L} \text {. }
$$

The stability domain $S$ follows from solving $\left|P\left(-s+r \mathrm{e}^{\mathrm{i} \mu}\right)\right|=1$ for $r$, while varying $\mu$, and is given in Fig. 3. The value $s$ should be chosen such that the straight line $z=-s+r \mathrm{e}^{\mathrm{i} \mu}$ for every fixed $\mu$ and varying $r$ intersects the upper half of $\partial S$ at only one point and does not come close to being tangent to $\partial S$, in order to make the equation for $r$ easy to solve numerically; $s=2$ is found to be suitable. $S$ is covered to a satisfactory extent by the ellipse (3.17) with $a=2.7853, b=2.55$. Stability conditions follow from Theorem 3.3. It seems that stability conditions for Runge-Kutta schemes applied to the convection-diffusion equation have been given in special cases only, such as for $d=0$ (zero diffusion coefficient) and $\kappa=1$ (second-order central scheme).

Leapfrog-Euler This is an example of a mixed scheme, in which different time discretizations are used for the convection and diffusion terms. Leapfrog is applied to the convection term, and explicit Euler to the diffusion term. The stability polynomial is given by

$$
\xi^{2}+2 \gamma(\theta) \xi+2 \delta(\theta)-1
$$

with $\gamma=\gamma_{1}+\mathrm{i} \gamma_{2}$. For mixed methods, the coefficients of the stability polynomial and hence the location of its roots do not depend on a single complex parameter $z(z=\gamma+\delta$ in the preceding cases), so that the stability domain $S$ is no longer a subset of the complex plane. In the case of (4.6), where $\delta$ is real, the roots depend on three parameters, $\gamma_{1}, \gamma_{2}$ and $\delta$, and $S$ is a subset of $\mathbb{R}^{3}$. It would not be difficult to reinterpret our theorems in this three-dimensional setting, but a visual check whether the sets of the theorems are contained in $S$ is much less straightforward in three than in two dimensions. We will not do this here, and restrict ourselves for mixed schemes to the case $\gamma_{1}=0$, i.e. central second-order 


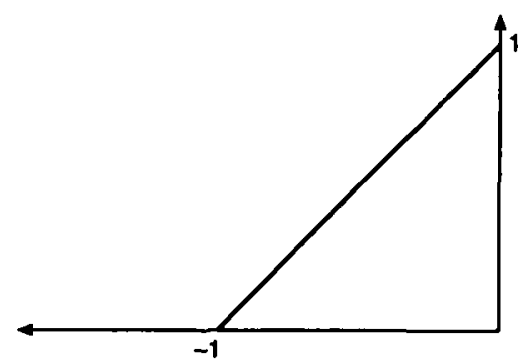

Fıg. 4. Stability domain of the leapfrog-Euler scheme.

$(\kappa=1)$ or fourth-order (scheme 2) discretization of the convection term. Hence, the roots of the stability polynomial depend only on two parameters, $\delta$ and $\gamma_{2}$, and we can continue to work in the complex $z$-plane, with $z=-\delta-i \gamma_{2}$.

Substitution of $\xi=\mathrm{e}^{\mathrm{i} \mu}, 0 \leqslant \mu<2 \pi$, in (4.6), equating the stability polynomial to zero and solving for $z=-\delta-\mathrm{i} \gamma_{2}$ as a function of $\mu$ gives the boundary of the stability domain $S$, the upper half of which is shown in Fig. 4. The ellipses, oval and parabola fit badly, and we proceed directly. For von Neumann stability it is necessary and sufficient that $\delta+\left|\gamma_{2}\right| \leqslant 1$, or, for $\kappa=1$,

$$
\sum_{\alpha}\left\{d_{\alpha}\left(1-\cos \theta_{\alpha}\right)+\left|c_{\alpha} \sin \theta_{\alpha}\right|\right\} \leqslant 1, \quad 0 \leqslant \theta_{\alpha}<2 \pi
$$

Since $\theta_{1}, \ldots, \theta_{m}$ are independent, the maximum is obtained by maximizing each term individually. Defining $f(\theta)=d(1-\cos \theta)+|c| \sin \theta, 0 \leqslant \theta<\pi$, we have $f^{\prime}(\theta)=0$ for $\theta=\pi-\gamma, \tan \gamma=|c| / d, 0 \leqslant \gamma<\pi / 2$, resulting in

$$
\max \{f(\theta): 0 \leqslant \theta<\pi\}=d+\sqrt{c^{2}+d^{2}} .
$$

This gives us the following necessary and sufficient stability condition:

$$
\sum_{\alpha}\left\{d_{\alpha}+\sqrt{d_{\alpha}^{2}+c_{\alpha}^{2}}\right\} \leqslant 1 .
$$

The one-dimensional version of this result has appeared in Chan (1984), with a less elementary proof (using Schur-Cohn theory). On heuristic grounds, the following stability condition, generally used in practice, was put forward in Schumann (1975) and proved in Pourquie (1994) (using Schur-Cohn theory):

$$
\sum_{\alpha}\left\{2 d_{\alpha}+\left|c_{\alpha}\right|\right\} \leqslant 1 \text {. }
$$

We see that this condition is sufficient but not necessary. Because of trouble with spurious modes, necessitating application of the so-called Asselin filter (Asselin (1972)), this scheme now seems to be less favoured than Adams-Bashforth for large-eddy and direct simulation of turbulence.

For fourth-order central discretization (2.5) (scheme 2) we must have:

$$
\sum_{\alpha}\left\{d_{\alpha}\left(1-\cos \theta_{\alpha}\right)+\frac{1}{6}\left|c_{\alpha}\right|\left|8 \sin \theta_{\alpha}-\sin 2 \theta_{\alpha}\right|\right\} \leqslant 1, \quad 0 \leqslant \theta_{\alpha}<2 \pi \text {. }
$$




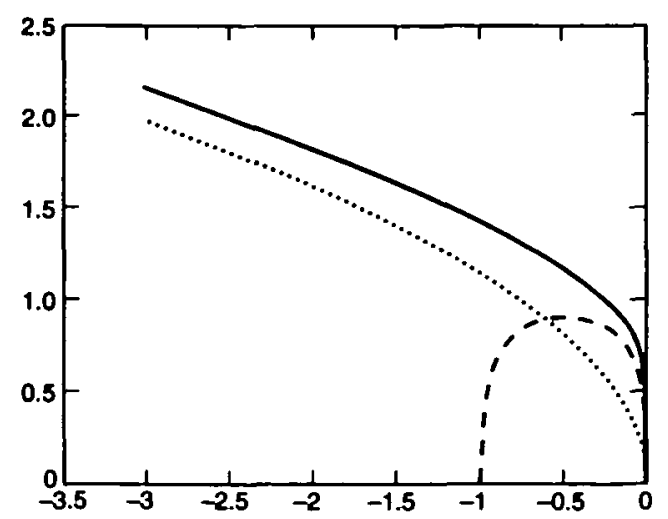

FIG. 5. Stability domain of the Adams-Bashforth-Crank-Nicolson scheme $(-)$ with oval $(-$,$) and$ parabola (...).

Since $\left|8 \sin \theta_{\alpha}-\sin 2 \theta_{\alpha}\right| \leqslant 10\left|\sin \theta_{\alpha}\right|$ it is sufficient if

$$
\sum_{\alpha}\left\{d_{\alpha}\left(1-\cos \theta_{\alpha}\right)+\frac{5}{3}\left|c_{\alpha} \sin \theta_{\alpha}\right|\right\} \leqslant 1, \quad 0 \leqslant \theta_{\alpha}<2 \pi .
$$

Proceeding as before we find the following sufficient condition:

$$
\sum_{\alpha}\left\{d_{\alpha}+\sqrt{d_{\alpha}^{2}+\frac{25}{9} c_{\alpha}^{2}}\right\} \leqslant 1
$$

Adams-Bashforth-Crank-Nicolson This is an example of a mixed scheme of IMEX (implicit-explicit) type. Second-order Adams-Bashforth is applied to the convection term and Crank-Nicolson to the diffusion term. The stability polynomial is given by

$$
\xi^{2}\left\{1+\frac{1}{2} \delta(\theta)\right\}+\xi\left\{\frac{1}{2} \delta(\theta)+\frac{3}{2} \gamma(\theta)-1\right\}-\frac{1}{2} \gamma(\theta)
$$

with $\gamma=\gamma_{1}+\mathrm{i} \gamma_{2}$. Again, this being a mixed scheme, the roots are not a function of $z=\delta+\gamma$, but our approach still works if $\gamma_{1}=0$, in a similar way as in the preceding case. The stability domain $S$ in the $z$-plane, $z=-\delta-\mathrm{i} \gamma_{2}$, is plotted in Fig. 5, together with the parabola (3.21) with $b=2 / \sqrt{3}$ and the oval (3.12) with $a=1 / 2, b=(3 / 4)^{1 / 4}$. We have (parabola $\cup$ oval) $\subseteq S$, so that for $\kappa=1$ (convection second-order central) Theorems 3.2 and 3.4 give the following sufficient stability conditions:

$$
\left\{d \leqslant 1 / 2 \text { and } \sum_{\alpha}\left(c_{\alpha}^{4} / d_{\alpha}\right)^{1 / 3} \leqslant(3 / 2)^{1 / 3}\right\} \quad \text { or } \quad \sum_{\alpha} c_{\alpha}^{2} / d_{\alpha} \leqslant 2 / 3
$$

whereas for scheme 2 Theorems 3.4 and 3.5 give

$$
\left\{d \leqslant 1 / 2 \quad \text { and } \quad \sum_{\alpha}\left(c_{\alpha}^{4} / d_{\alpha}\right)^{1 / 3} \leqslant q_{4}(3 / 2)^{1 / 3}\right\} \quad \text { or } \quad \sum_{\alpha} c_{\alpha}^{2} / d_{\alpha} \leqslant 3^{4} / 5^{3} .
$$




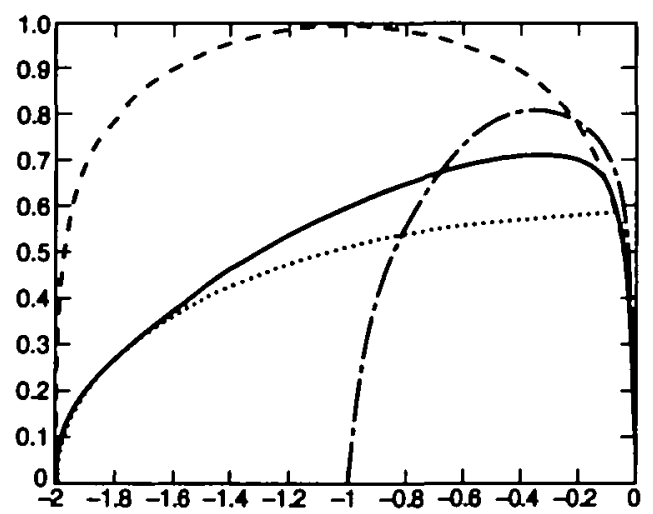

FIG. 6. Stability domains of Adams-Bashforth-Euler ( - ) and second-order Adams-Bashforth (-.-.), with oval (- - ) and osculating ellipse ( . - ).

Using Schur-Cohn theory, in Varah (1980) the following sufficient condition is derived for the one-dimensional case and $\kappa=1: c_{1}^{2} / d_{1} \leqslant 1 / 6$, which is significantly more restrictive than (4.14); a symptom of the unwieldiness of the Schur-Cohn conditions.

Adams-Bashforth-Euler Like Adams-Bashforth, this is another scheme that tends to replace leapfrog-Euler. The convection term is treated with secondorder Adams-Bashforth and the diffusion term with explicit Euler. The stability polynomial is given by

$$
\xi^{2}-\xi+\delta(\theta) \xi+\frac{1}{2} \gamma(\theta)(3 \xi-1)
$$

with $\gamma=\gamma_{1}+\mathrm{i} \gamma_{2}$, and again our method works only if $\gamma_{1}=0$. The stability domain $S$ in the $z$-plane, $z=\delta+\mathrm{i} \gamma_{2}$, is plotted in Fig. 6. For comparison, the stability domain of second-order Adams-Bashforth is also shown. The ellipse (3.17) that osculates in $z=-2$ has parameters $a=2, b=1 / \sqrt{3}$. The oval in Fig. 6 has parameters $a=b=1$. We have \{ellipse $\cap$ oval\} $\subseteq S$, so that for $\kappa=1$, Theorems 3.2 and 3.3 give

$$
d \leqslant 1 \quad \sum_{\alpha}\left(c_{\alpha}^{4} / d_{\alpha}\right)^{1 / 3} \leqslant 1 \quad d \sum_{\alpha} c_{\alpha}^{2} / d_{\alpha} \leqslant \frac{1}{6}\left(1+\sqrt{1-d^{2}}\right)
$$

whereas for scheme 2, Theorems 3.5 and 3.3 give, by substituting $k=1 / 3$ and $\bar{c}_{\alpha}=0$ (cf. the remark before Theorem 3.5),

$$
d \leqslant 1 \quad \sum_{\alpha}\left(c_{\alpha}^{4} / d_{\alpha}\right)^{1 / 3} \leqslant q \quad d \sum_{\alpha} c_{\alpha}^{2} / d_{\alpha} \leqslant \frac{3}{50}\left(1+\sqrt{1-d^{2}}\right)
$$

with

$$
q=\frac{1}{5}\left(\frac{5+\sqrt{10}}{12}\right)^{1 / 3}(\sqrt{10}-1)^{5 / 3} \cong 0.6360
$$




\section{Concluding remarks}

von Neumann stability analysis for the convection-diffusion equation involves deriving conditions for the roots of the characteristic polynomial of the multistep time discretization method employed to be in the unit disk. Schur-Cohn theory, as described in Miller (1971), gives necessary and sufficient conditions on the coefficients. However, deriving stability restrictions on the time step from these conditions is usually an arduous task, that has to be undertaken anew for each scheme that one wishes to consider, and gets rapidly out of hand as the order of the multistep method increases. Hence, it is not surprising that few results have been published. Furthermore, Schur-Cohn theory is not applicable to Runge-Kutta methods, for which the characteristic polynomial itself is not to exceed 1, rather than the absolute value of the roots. In the foregoing we have presented an alternative approach. Based on Theorems 3.1-3.5, stability conditions are easy to find. Our approach does not get more complicated as the order of multistep methods increases, and applies equally to Runge-Kutta methods. Although the basic principle is not affected, its ease of use in practice is if the characteristic polynomial depends on more than two parameters, so that the classical stability diagram of the time discretization method does not apply. This may happen if the terms in the partial differential equation under consideration are not all discretized in time by the same scheme, i.e. with hybrid schemes, of which IMEX schemes are a subclass. Nevertheless, for such schemes the method may still work, for example, when central space discretization is applied to first-order terms.

The principle of the method is applicable to general initial boundary value problems in any number of dimensions, but Theorems 3.1-3.5 have been derived for the convection-diffusion equation, with the $x$-scheme or the fourth-order central scheme used for space discretization of the convection term. To illustrate the use of the method, von Neumann stability conditions are derived for a number of schemes. In most cases, sufficient conditions do not seem to have been available before.

\section{Acknowledgement}

The author is indebted to $\mathrm{K}$. Dekker for useful discussions.

\section{REFERENCES}

ANDERSON, W. K., ThOMAS, J. L., \& VAN LEER B 1985 A comparison of finite volume flux vector splittings for the Euler equations. AlAA Paper 85-0122, American Institute of Aeronautics and Astronautics, New York.

AsSELIN, R. 1972 Frequency filter for time integrations. Mon. Weather Rev. 100, 487-90.

CHAN, T. F. 1984 Stability analysis of finite difference schemes for the advection-diffusion equation. SIAM J. Numer. Anal. 21, 272-84.

CHORIN, A. J. 1968 Numerical solution of the Navier-Stokes equations. Math. Comput. 22, 745-62.

FromM, J. E. 1968 A method for reducing dispersion in convective difference schemes. J. Comput. Phys. 3, 176-89. 
Hindmarsh, A. C., Gresho, P. M., \& Griffiths, D. F. 1984 The stability of explicit Euler time-integration for certain finite difference approximations of the multi-dimensional advection-diffusion equation. Int. J. Numer. Meth. Fluids 4, 853-97.

HIRT, C. W. 1968 Heuristic stability theory for finite difference equations. J. Comput. Phys. 2, 339-55.

LEONARD, B. P. 1979 A stable and accurate convective modelling procedure based on quadratic upstream interpolation. Comput. Meth. Appl. Mech. Eng. 19, 59-98.

MiLLER, J. J. H. 1971 On the location of zeros of certain classes of polynomials with applications to numerical analysis. J. Inst. Math. Appl. 8, 397-406.

Morton, K. W. 1971 Stability and convergence in fluid flow problems. Proc. R. Soc. London A 323, 237-53.

POURQuIE, M. J. B. M. 1994 Large-eddy simulation of a turbulent jet. PhD Thesis, Delft University of Technology.

SCHUMANN, U. 1975 Linear stability of finite difference equations for three-dimensional flow problems. J. Comput. Phys. 18, 465-70.

SOMMEIJER, B. P., VAN DER HOUWEN, P. J., \& KOK, J. 1994 Time integration of three-dimensional numerical transport models. Appl. Numer. Math. 16, 201-25.

STEGER, J. L., \& WARMING, R. F. 1981 Flux-vector splitting of the inviscid gas-dynamic equations with applications to finite-difference methods. J. Comput. Phys. 32, 263-93.

VARAH, J. M. 1980 Stability restrictions on second order, three level finite difference schemes for parabolic equations. SIAM J. Numer. Anal. 17, 300-9.

VAN KAN, J. J. I. M. 1986 A second-order accurate pressure correction method for viscous incompressible flow. SIAM J. Sci. Stat. Comput. 7, 870-91.

VAN LEER, B. 1985 Upwind-difference methods for aerodynamic problems governed by the Euler equations. Lectures Appl. Math. 22, 327-36.

WESSELING, P. 1973 On the construction of accurate difference schemes for hyperbolic partial differential equations. J. Eng. Math. 7, 1-31.

Wesseling, P. 1995 A method to obtain von Neumann stability conditions for the convection-diffusion equation. Numerical Methods for Fluid Dynamics V, Proc. ICFD Conf. on Numerical Methods in Fluid Dynamics (Oxford, 3-6 April 1995) (M. J. Baines and K. W. Morton, eds). Oxford: Oxford University Press, pp 211-224. 